\title{
Growth Arrest of Individual Senile Plaques in a Model of Alzheimer's Disease Observed by In Vivo Multiphoton Microscopy
}

\author{
R. H. Christie, ${ }^{1}$ B. J. Bacskai, ${ }^{1}$ W. R. Zipfel, ${ }^{2}$ R. M. Williams, ${ }^{2}$ S. T. Kajdasz, ${ }^{1}$ W. W. Webb, ${ }^{2}$ and B. T. Hyman ${ }^{1}$ \\ ${ }^{1}$ Alzheimer's Disease Research Unit, Massachusetts General Hospital, Charlestown, Massachusetts 02129, and 2School \\ of Applied and Engineering Physics, Cornell University, Ithaca, New York 14853
}

In Alzheimer's disease, amyloid- $\beta$ peptide aggregates in the extracellular space to form senile plaques. The process of plaque deposition and growth has been modeled on the basis of in vitro experiments in ways that lead to divergent predictions: either a diffusion-limited growth model in which plaques grow by first-order kinetics, or a dynamic model of continual deposition and asymmetrical clearance in which plaques reach a stable size and stop growing but evolve morphologically over time. The models have not been tested in vivo because plaques are too small (by several orders of magnitude) for conventional imaging modalities. We now report in vivo multiphoton laser scanning imaging of thioflavine S-stained senile plaques in the Tg2576 transgenic mouse model of Alzheimer's disease to test these biophysical models and show that there is no detectable change in plaque size over extended periods of time. Qualitatively, geometric features remain unchanged over time in the vast majority of the 349 plaques imaged and re-imaged. Intervals as long as 5 months were obtained. Nonetheless, rare examples of growth or shrinkage of individual plaques do occur, and new plaques appear between imaging sessions. These results indicate that thioflavine S-positive plaques appear and then are stable, supporting a dynamic feedback model of plaque growth.

Key words: amyloid; transgenic; Alzheimer; two-photon; in vivo imaging; senile plaque; microglia
Aggregates of the amyloid- $\beta$ peptide $(\mathrm{A} \beta)$ form senile plaques, one of the classic neuropathological lesions of Alzheimer's disease. In vitro observations show that $\mathrm{A} \beta$ is extremely insoluble and precipitates to form aggregates (Hilbich et al., 1991), and that exogenous $A \beta$ decorates existing plaques and enlarges them according to first-order kinetics (Jarrett and Lansbury, 1993; Esler et al., 1996). These observations led to a model in which a nidus is formed and plaques then grow in a time- and concentrationdependent manner (Jarrett and Lansbury, 1993; Esler et al., 1996).

Paradoxically, however, the size distribution of senile plaques appears to remain constant rather than increasing with increasing duration of illness (Hyman et al., 1993). Together with observations on the fine structure of plaques, these data led to a statistical physics-based dynamic feedback model in which a feedback process was postulated to limit plaque growth, leading to stable plaque size and, in principle, the shrinkage of existing plaques (Hyman et al., 1995; Cruz et al., 1997; Urbanc et al., 1999a,b). Distinguishing between these possibilities is important for understanding the life history and pathophysiology of amyloid deposition in Alzheimer's disease, but it has not been possible because direct measurements of plaques in vivo have not been achieved.

Tg2576 transgenic mice overexpress a mutant form of the human amyloid precursor protein and develop senile plaques in an age-related fashion, with amyloid- $\beta$ deposits occurring first at 8-10 months of age (Hsiao et al., 1996; Irizarry et al., 1997).

Received Aug. 14, 2000; revised Nov. 3, 2000; accepted Nov. 13, 2000.

This work was supported by National Institute on Aging Grants AG08487, P01 AG15453, and T32GM07753, as well as generous support from the Walters Family Foundation.

Correspondence should be addressed to Dr. Bradley Hyman, Alzheimer's Disease Research Unit, CNY 6405, Massachusetts General Hospital, 149 13th Street, Charlestown, MA 02129. E-mail: B_Hyman@helix.mgh.harvard.edu.

Copyright (C) 2001 Society for Neuroscience 0270-6474/01/210858-07\$15.00/0
The number of plaques increases dramatically with age. Here we develop the successful imaging of senile plaques in living animals using in vivo multiphoton laser scanning microscopy through an intact skull window, which allows us to image and re-image the same brain region many times over a period of days to months. In this study, we also introduce the use of multiphoton imaging for chronic, in vivo brain imaging. Multiphoton excitation is based on the simultaneous absorption of multiple low-energy photons; the sum of the energies of these photons is sufficient to excite fluorescence in an appropriate fluorophore. Relatively benign longwavelength light generates fluorescence that would otherwise require potentially damaging levels of ultraviolet radiation. Another important benefit is that the infrared wavelengths penetrate tissue without absorption by blood and with less scattering than visible light, allowing imaging deeper into the brain. Multiphoton excitation is achieved by focusing a sub-picosecond pulsed laser into the sample through a microscope objective. The higher-order power dependence of multiphoton excitation results in a restricted excitation volume enabling spatial resolution of $\sim 1 \mu \mathrm{m}$, essentially equivalent to that of confocal microscopy even in turbid tissue (Denk et al., 1990). The resulting elimination of out-of-plane free radical generation and photobleaching are additional advantages for in vivo applications.

For the first application of this new technology, we examined the natural history of individual senile plaques in the brains of living transgenic mice. We conclude that individual plaques achieve and maintain a stable size in accord with the predictions of the dynamic feedback model.

\section{MATERIALS AND METHODS}

In vivo imaging of amyloid deposition. Nine male $\mathrm{Tg} 2576$ mice (mean age 18.6 months) (Hsiao et al., 1996) were used for the in vivo imaging of plaques. These mice express the human amyloid precursor protein carrying the Swedish mutation under the hamster prion protein promoter. 
The skull was prepared 2-6 d before imaging. Mice were anesthetized with avertin (tribromoethanol; $250 \mathrm{mg} / \mathrm{kg}$, i.p.). A high-speed drill (Fine Science Tools, Foster City, CA) was used to thin each skull in a circular region $\sim 1-1.2 \mathrm{~mm}$ in diameter (see Fig. $2 a$ ), and a dissecting microscope (Leica, Wetzlar, Germany) was used for gross visualization of the site. Heat and vibration artifacts were minimized during drilling by frequent application of artificial CSF (ACSF) containing (in mM): $125 \mathrm{NaCl}, 26$ $\mathrm{NaHCO}_{3}, 1.25 \mathrm{NaH}_{2} \mathrm{PO}_{4}, 2.5 \mathrm{KCl}, 1 \mathrm{MgCl}_{2}, 1 \mathrm{CaCl}_{2}$, and 25 glucose. Skull thickness was repeatedly assessed with a surgical probe (Roboz, Rockville MD), and drilling stopped when the bone displayed flexibility in a central region $\sim 0.6 \mathrm{~mm}$ in diameter. Clear visualization of pial vasculature was an additional indication of skull thinness. The scalp was then sutured, and the animal allowed to recover. On the day of imaging, the animal was re-anesthetized, the scalp was reflected, and the small amount of connective tissue that had grown in the interim was removed by scraping. The tip of a 22 gauge needle was used to make a small break in the lateral wall of the skull preparation to facilitate thioflavine $\mathrm{S}$ diffusion into the brain. Thioflavine S $(0.005 \%$ in ACSF) (Sigma, St. Louis, MO) was then applied for $20 \mathrm{~min}$ to the site. A small ring of molten bone wax was applied to the skull surrounding the site, and this well was filled with ACSF to create an aqueous reservoir for the long working distance, water immersion dipping objectives (Olympus, Tokyo, Japan). The thin-skull preparation also eliminates the need for application of a coverslip (Svoboda et al., 1997) to the imaging site because preservation of this thin layer of bone is sufficient to stabilize the cardiac and respiratory motion of the brain inherent in in vivo imaging. The animal was immobilized in custom-built stage-mounted ear bars and a nosepiece, similar in design to a stereotaxic apparatus. The thin-skull site was then placed directly under the objective lens of the microscope (Olympus BX-50) for imaging (see Fig. 1b).

Two-photon fluorescence was generated with $750 \mathrm{~nm}$ excitation from a mode-locked Ti:Sapphire laser [Tsunami (Spectra-Physics, Mountain View, CA), $5.45 \mathrm{~W}$ Millenium V pump laser (Spectra-Physics), power at back aperture of objective $10 \mathrm{~mW}$, pulse $60-100 \mathrm{fs}$ ] mounted on a commercially available multiphoton imaging system (Bio-Rad 1024ES; Bio-Rad, Hercules, CA). Custom-built external detectors containing three photomultiplier tubes (Hamamatsu Photonics, Bridgewater, NJ) collected emitted light in the range of $380-480,500-540$, and 560-650 $\mathrm{nm}$; all thioflavine S figures are from the $380-480 \mathrm{~nm}$ channel. Imaging was performed using the normal scan speed of the scanhead, dwell time $=1.5 \mu$ sec per pixel. Up to four thin-skull preparations were made per animal to maximize the number of plaques available for measurement. Thioflavine S $(0.005 \%$ in ACSF) was applied to the preparation at each imaging session. The site was first imaged with a $10 \times$ objective $(1230 \mathrm{~mm}$ square field; NA $=0.5)$ to map the surface of the thin-skull preparation. This low-power map, in conjunction with orienting markings on the microscope stage and stereotaxic frame, allowed for precise repositioning of the site under the microscope objective during subsequent imaging sessions. The $x-y$ stage encoders (Boeckeler, Tucson, AZ) were calibrated with their origin at the center of the thin-skull site and were used to preserve the relative coordinates of higher-magnification images within the site. Nine $z$-series using a $60 \times$ objective $(205 \times 205$ $\mu \mathrm{m} ; \mathrm{NA}=0.8$ ) were then collected in a $3 \times 3$ array covering the thinnest portion of the site by moving the stage exactly $205 \mu \mathrm{m}$ in the $x$ or $y$ direction. The incremental $z$-step was $2 \mu \mathrm{m}$, and the series was collected from the skull surface to a depth of $\sim 150 \mu \mathrm{m}$ into the brain. The starting position of the $z$-axis motor relative to skull position was recorded for later $z$-axis alignment during montage generation. After completion of image collection, the animal was removed from the stage, the ring of bone wax was removed, the skull was washed with sterile saline, and the scalp was sutured. The animal was warmed to $37^{\circ} \mathrm{C}$ during recovery from anesthesia. Total time of anesthesia was limited to $2 \mathrm{hr}$.

Image analysis. Montages were reconstructed into a single stack of images using Scion Image (Scion, Frederick, MD). The area of individual plaque cross sections was measured in each optical section by thresholding at $2 \mathrm{SD}$ above the mean of an adjacent background region. Plaques that did not satisfy the criteria of imaging were eliminated from the measurement set. Plaques on the edge of the imaging area or on one of the montage lines were rejected because of the potential imprecision of moving the animal on the stage. Plaques for which the intensity was not sufficiently above the background for appropriate thresholding were also eliminated. This rejected many plaques, typically deep in the preparation, that appeared to be present but were too faint to measure using the automatic threshold technique. Finally, plaques with images that contained any appreciable motion artifact were rejected. Of the 448 plaques
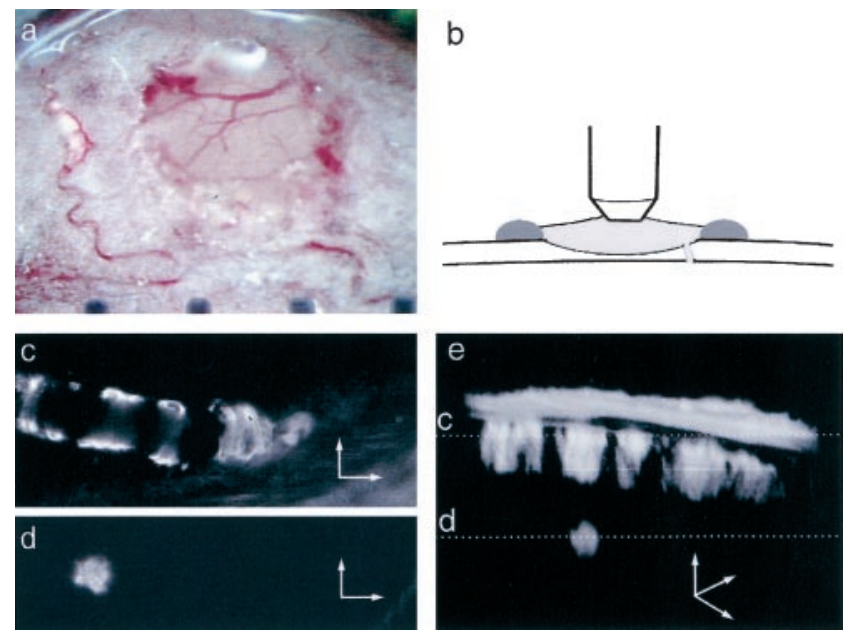

Figure 1. Preparation of skull for in vivo imaging. $a$, Gross appearance of skull through dissecting microscope before imaging. The pial vasculature is visible through the intact but thinned region of skull. Anterior midline sutures are also visible in the image. Scale marks are spaced $1 \mathrm{~mm}$ apart. $b$, Schematic diagram of the microscope objective during imaging. The thinned area of skull is bathed in a pool of ACSF (light gray) that is retained by a ring of bone wax (dark gray). A small break is made in the lateral wall of the thinned area to allow for thioflavine $\mathrm{S}$ entry. $c$, In vivo visualization of thioflavine S-positive amyloid in a 15-month-old Tg2576 mouse. A single optical section near the surface of the skull is shown. Thioflavine S-positive amyloid angiopathy is visible ringing the pial arteriole in this image. The fainter autofluorescence of the skull bone is visible in the bottom right corner; the fibrous autofluorescence of the dura is visible as a band at bottom right. $d$, Another optical section from the same $z$-series as $c$, but $50 \mu \mathrm{m}$ deeper into the brain, showing a thioflavine $\mathrm{S}$-positive amyloid deposit in layer 1 of the mouse cortex. $e$, Perpendicular volume rendering of the entire stack of images, with the skull visible at the top, the amyloid-encrusted pial vessel just beneath, and the thioflavine S-positive plaque deep in the living brain. The autofluorescent dura can also be seen as a faint layer between the vessel and the skull. The approximate levels of optical sections shown in $c$ and $d$ are represented by dotted lines. Scale bars, $25 \mu \mathrm{m}$.

imaged, 349 met these criteria. Maximal plaque diameter was then calculated from the cross section of the largest area for each plaque. Volume rendering was performed using VoxBlast (VayTek, Fairfield, IA) on a Windows NT-based workstation (Precision 610; Dell Computer, Round Rock, TX).

Angiography. The tail of the animal was warmed on a heating pad to dilate the blood vessels, and $\sim 0.05 \mathrm{ml}$ of fluorescein $(25 \mathrm{mg} / \mathrm{ml})$ in sterile PBS was injected into a tail vein of the mouse at least 20 min before imaging. The dye did not cross the blood-brain barrier and permitted concurrent visualization of blood vessels throughout the imaging volume in the brain.

Histology. Two groups of animals ( $n=3$ per group; mean ages 12.6 and 22.6 months) were used for the histological measurement of amyloid deposition, measuring amyloid burden and size distribution as previously described (Hyman et al., 1993). Images of thioflavine S-stained sections were collected using two-photon excitation with $750 \mathrm{~nm}$ light. All fields of the cortex containing thioflavine S-positive amyloid deposits were imaged in a given section until $\sim 80$ plaques were imaged per animal. Images were then transferred to Scion Image (Scion), where a threshold was applied, the image was filtered slightly to remove noise, and the plaques were automatically outlined by the particle analysis protocol of the software. Images were manually edited to remove thioflavine S-positive blood vessels and edge-effect artifacts. Sections containing few thioflavine S-positive plaques were exhaustively sampled, and all plaques within this cortical area were counted. Random systematic sampling of $\sim 10$ fields per section was applied to those sections containing heavier amyloid burdens, and a $400 \times 400 \mu \mathrm{m}$ counting frame was used with automatic selection and measurement to count thioflavine S-positive plaques. Plaques were counted in three sections per animal in this way; the adequacy of the sampling strategy was reflected in coefficients of 
error $\leq 10 \%$. Results were expressed as the density of thioflavine S-positive plaques per square millimeter. Statistical significance of the observed difference in plaque number between the groups was assessed by $t$ test.

For immunostaining of intact brain to detect $\mathrm{A} \beta$, monoclonal anti-A $\beta$ antibody 10D5 (Elan Pharmaceuticals, South San Francisco, CA) was directly conjugated to $\mathrm{Cy} 3$ using a commercially available kit (CyDye; Amersham Pharmacia, Piscataway, NJ) and applied in conjunction with thioflavine $\mathrm{S}(0.005 \%$ in $\mathrm{ACSF})$ to the surface of a fixed, unsectioned brain for $20 \mathrm{~min}$.

\section{RESULTS}

We adapted multiphoton microscopy for these studies because it has unique advantages for in vivo imaging and its resolution is on the order of $1 \mu \mathrm{m}$ (Denk et al., 1990). Because only acute in vivo imaging has been reported to date in any system, we developed a new approach for long-term repeat imaging. A thin, transparent bone window $\sim 1 \mathrm{~mm}$ in diameter and $\sim 20 \mu \mathrm{m}$ thick is formed with a high-speed burr in the skull of an anesthetized Tg2576 mouse (Fig. 1a). A small break is made in the lateral wall of the site to allow for delivery of fluorophore to the brain, but the bone remains otherwise intact within the thinned region. An upright Olympus BX-50 fixed-stage microscope containing a modified stage insert was used for in vivo imaging (Fig. 1b).

Thioflavine $\mathrm{S}$ is a standard amyloid-binding fluorophore that excites in the ultraviolet range and has an emission maximum of $\sim 450 \mathrm{~nm}$. It has been extensively used to label amyloid deposits in human Alzheimer's disease tissue (Kelenyi, 1967) as well as in transgenic mouse models of amyloid deposition. It is among the stains recommended by the Consortium to Establish a Registry for Alzheimer's Disease for the neuropathological diagnosis of Alzheimer's disease in postmortem tissue (Mirra et al., 1991). A dilute solution of thioflavine $\mathrm{S}$ was applied to the brain of a living 18-month-old $\mathrm{Tg} 2576$ transgenic mouse for in vivo visualization of amyloid deposits using multiphoton microscopy. Optical sections were obtained every $2.0 \mu \mathrm{m}$, from the bone window surface to $\sim 150 \mu \mathrm{m}$ beneath the surface, using $750 \mathrm{~nm}$ light for two-photon excitation of the fluorophore. Reconstruction of these thin optical sections revealed thioflavine S-positive amyloid surrounding pial arterioles with the classic segmental appearance of amyloid angiopathy (Vonsattel et al., 1991) in superficial sections (Fig. 1c). Deeper optical sections (Fig. $1 d$ ) revealed parenchymal thioflavine $\mathrm{S}$-positive amyloid plaques. Plaques were visualized in this way up to $150 \mu \mathrm{m}$ beneath the surface of the cortex.

The imaged plaques share the morphology of classic thioflavine S-positive senile plaques seen in tissue from transgenic animals and from Alzheimer's disease cases, and no such structures were seen in nontransgenic control littermates. That these structures are indeed senile plaques was further confirmed by incubation of the postmortem fixed brain from the transgenic mouse with a fluorescently labeled antibody to $\mathrm{A} \beta$ (10D5; Elan Pharmaceuticals) (Hyman et al., 1992) directly labeled with Cy3 (Amersham). This double stain revealed colocalization of thioflavine $\mathrm{S}$ with surrounding amyloid- $\beta$ immunoreactivity (Fig. 2a,b). As expected, plaques that have a dense core are stained by thioflavine $\mathrm{S}$ and are a subset of all $\mathrm{A} \beta$ immunoreactive structures (Schmidt et al., 1995). Moreover, histological analysis 2-7 d after such imaging reveals no overt damage, neuronal loss, or increase in reactive astrocytes (Fig. 2c) as assessed by glial fibrillary acidic protein staining, suggesting that the thin-skull preparation and imaging protocol are well tolerated by the living brain.

The potential of multiphoton microscopy for nondestructive in vivo imaging opens the possibility of repeated visualization of plaques over time within a living animal. Figure $3 a$ is an example
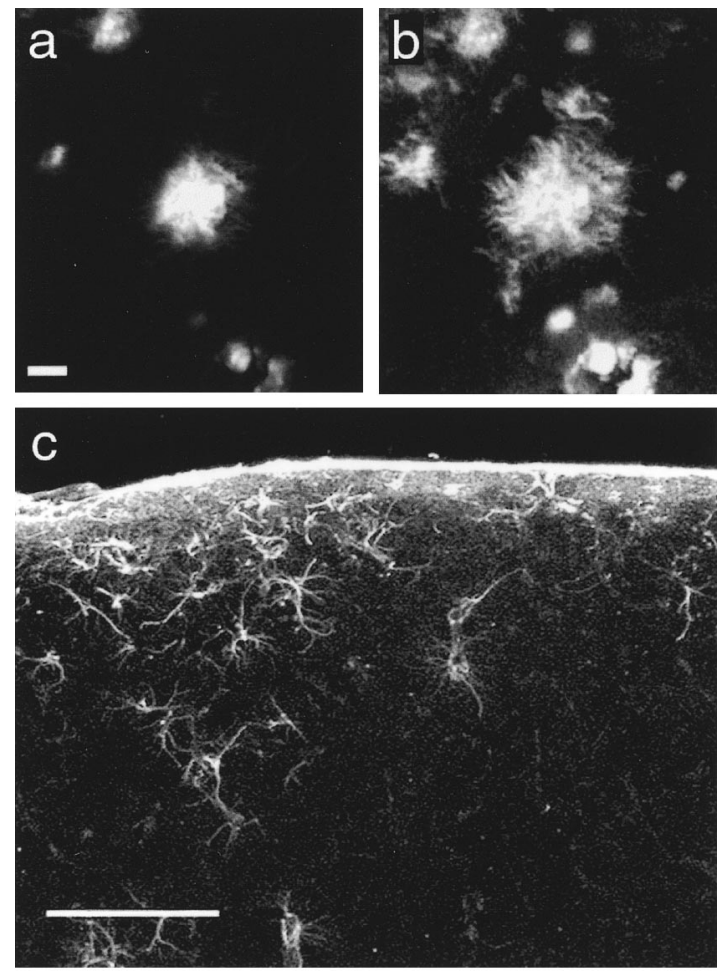

Figure 2. To confirm that the thioflavine S-positive structures were indeed senile plaques, thioflavine $S$ and an anti-amyloid- $\beta$ monoclonal antibody, cy3-labeled 10D5 (Elan Pharmaceuticals), were applied to the surface of a fixed but intact Tg2576 brain. $a$, Fluorescence emission in the range 380-480 $\mathrm{nm}$ shows thioflavine $\mathrm{S}$ staining the amyloid core of a plaque $\sim 40 \mu \mathrm{m}$ deep into the brain. Scale bar, $10 \mu \mathrm{m}$. $b$, Emission in the 560-650 nm range shows the Cy3-10D5 staining of the same A $\beta$ surrounding the thioflavine S-positive core. $c$, Glial fibrillary acidic protein immunoreactivity in a section through the area imaged by multiphoton microscopy 2 d previously. Sparse immunoreactive astrocytes, not substantially different from adjacent (nonimaged) cortex, suggest minimal tissue response to imaging. Scale bar, $100 \mu \mathrm{m}$.

of the imaging approach in a live mouse. The skull was prepared, thioflavine $\mathrm{S}$ was applied, and a $3 \times 3$ matrix of a $615 \times 615 \mu \mathrm{m}$ region of the site was imaged using a $60 \times$ water immersion objective. On recovery from anesthesia, the animal was returned to its cage where it showed no sign of impairment or discomfort after imaging. Representative images from one animal collected at an interval of $2 \mathrm{~d}$ are shown in Figure $3 b-e$. Examples of a plaque (Fig. 3b) and amyloid angiopathy (Fig. 3d) are shown at the initial imaging session. Two days later, the animal was reanesthetized, and thioflavine $S$ was reapplied to the thinned region of the skull. Imaging was performed under the same conditions as the initial session. Plaques (Fig. 3c) and amyloid angiopathy (Fig. 3e) were both clearly revisualized after $2 \mathrm{~d}$ and appear to have been unaltered since the initial imaging session.

To study the natural history of thioflavine S-positive amyloid deposits, plaques in eight additional animals (mean age 18.6 months) were imaged over progressively longer time periods. Variation in the thin-skull preparation prevented revisualization of the entire imaging volume from one imaging session to the next; the number of plaques within the imaging site could therefore not be counted in a statistically unbiased manner. A total of 41 imaging sessions yielded 29 data sets containing plaques that were successfully imaged more than once. The data sets contained 349 aligned pairs of plaques over extended time intervals; 

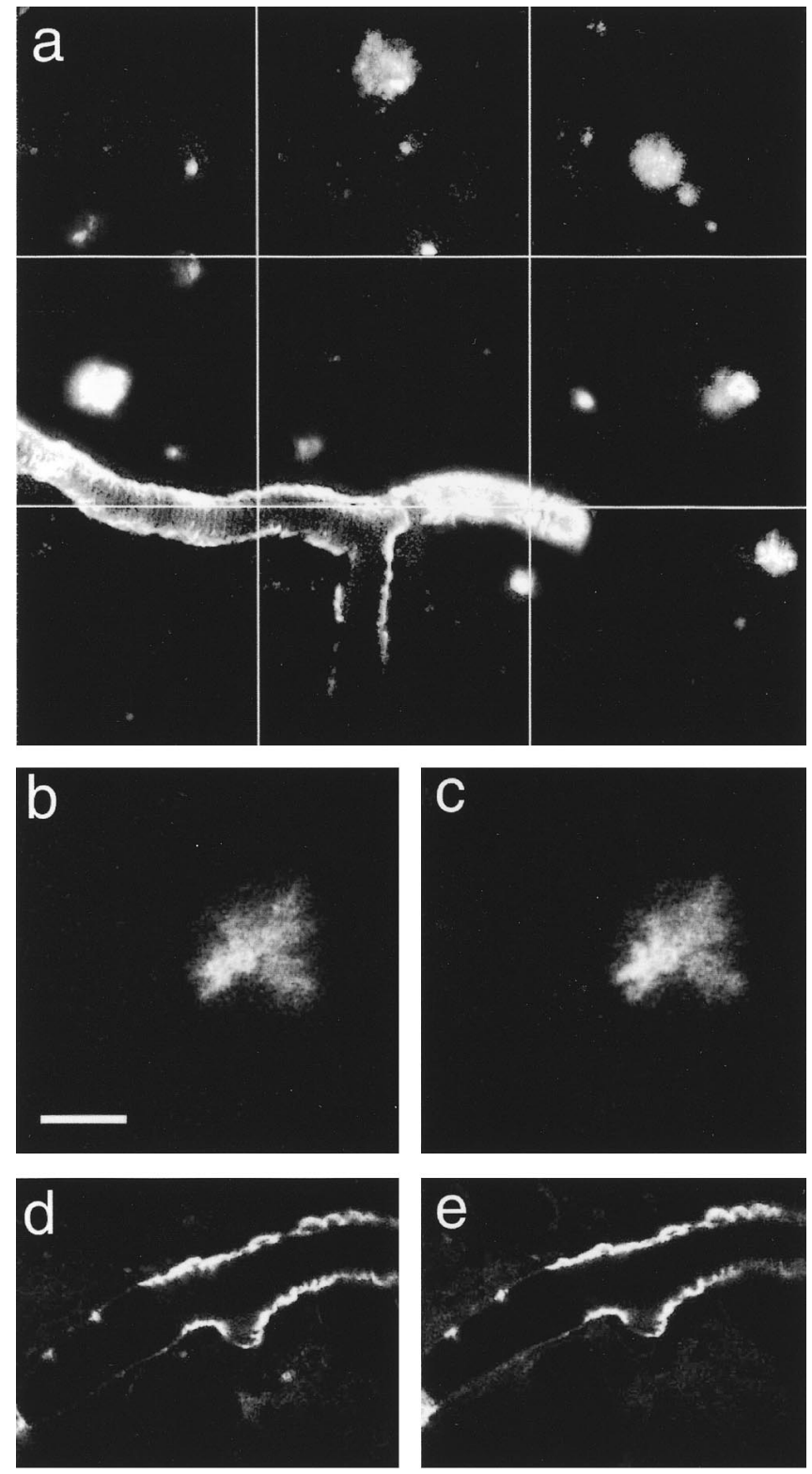

Figure 3. In vivo imaging of thioflavine S-positive amyloid deposition in a $\operatorname{Tg} 2576$ mouse. $a$, A $3 \times 3$ montage of $60 \times$ fields acquired on initial imaging day. Optical sections were obtained every $2 \mu \mathrm{m}$ for a distance of $200 \mu \mathrm{m}$ from the inner surface of the skull; images were aligned in the $x$, $y$, and $z$ axes, then projected onto a single image revealing amyloid angiopathy and senile plaques. $b$, In vivo imaging of a thioflavine S-positive plaque $\sim 40 \mu \mathrm{m}$ beneath the skull surface. This image is a single optical section through the body of the plaque. Scale bar, $10 \mu \mathrm{m} . c$, The same plaque as in $b$, re-imaged $2 \mathrm{~d}$ later under identical imaging conditions. $d$, Single optical section showing thioflavine S-positive amyloid angiopathy associated with a pial arteriole. $e$, The same arteriole as $d$, imaged after $2 \mathrm{~d}$.

one animal was re-imaged $150 \mathrm{~d}$ after initial imaging. As many as five separate imaging sessions of the same volume were obtained in each animal. Qualitatively, the structure and size of the vast majority of plaques remain remarkably stable over these extended periods of observation. Fine details of the morphology of individual plaques are recognizable in subsequent images obtained months later, including finger-like appendages and small clusters of dense thioflavine S-positive amyloid (Fig. 4).

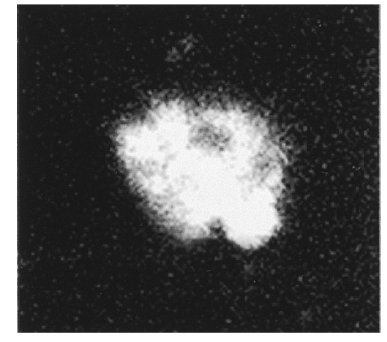

\section{Day}
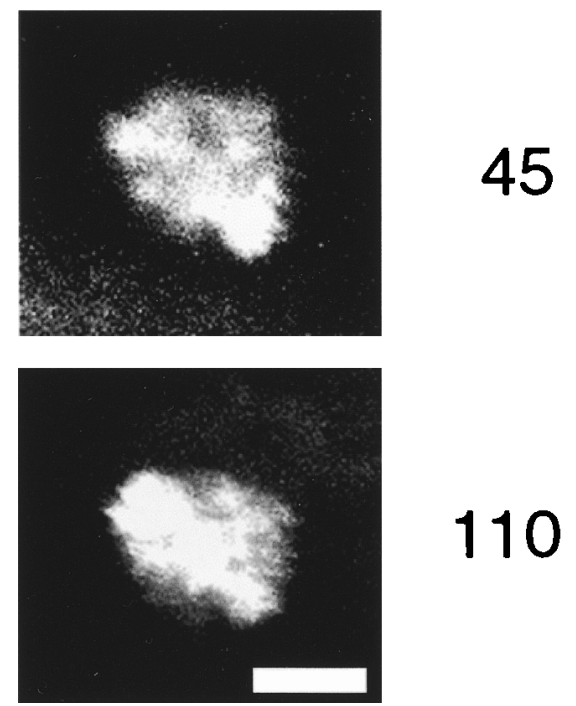

110

Figure 4. Sequential imaging of a plaque over time. Images of a single, identified plaque were obtained at the initial imaging session and again 45 and $110 \mathrm{~d}$ later in a live mouse. Fine details of the plaque are clearly recognizable over these ranges of time. Scale bar, $20 \mu \mathrm{m}$.

Quantitative analysis of plaque diameter over time, measured in the optical section with the greatest diameter, confirms this qualitative judgment. Analysis of changes in plaque diameter for the entire set of measurements is presented in Figure 5. The amount of variability in the two measures, taken as a population, is essentially the same, regardless of whether the measures were obtained 2 or $150 \mathrm{~d}$ apart (Fig. $5 a$ ). The initial measurement of the size of an individual plaque is an excellent predictor of a subsequent measurement of that same plaque, whether over an interval of days or months. The slope of the linear regression graph plotting the size of a plaque at initial imaging to its size at a later time, taking all measurements for periods over an interval of $2-150 \mathrm{~d}$, was nearly unity ( are consistent with plaques being extraordinarily stable in vivo objects over an extended period of time, and they suggest that individual plaques do not continue to grow.

Close examination of the data sets, however, reveals a small number of plaques that appear to have either grown or shrunk substantially between imaging sessions. We were concerned that this apparent change could be caused by technical factors, and so we systematically applied working criteria to eliminate known potential sources of measurement error. For example, we rejected data from plaques that fell on the border of one of the $205 \times 205$ $\mu \mathrm{m}$ fields comprising the montage and from the deepest plaque imaged in a given session. In several animals, fluorescein angiography was performed at the same time as thioflavine $\mathrm{S}$ imaging to create additional internal landmarks to facilitate lining up the 

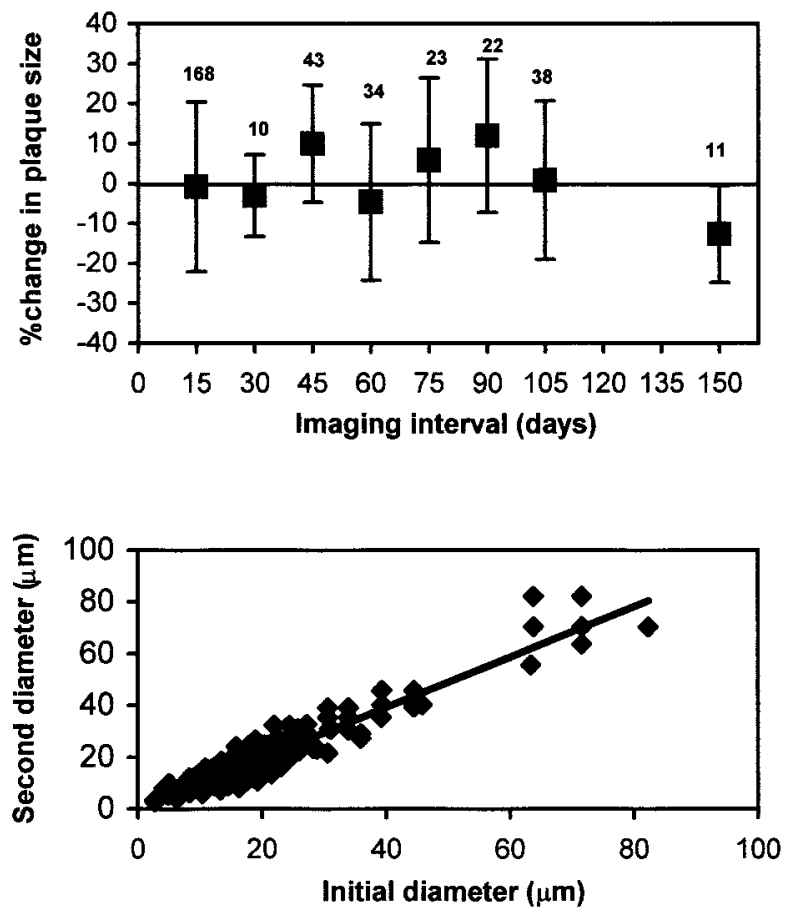

Figure 5. Analysis of variability of plaque measurements. Top, Percentage change (average \pm SD) for all plaque measurements binned into 0.5 month groups shows no trend in either the average measure or the variability of measurement over the time interval examined. $N$ values for each measurement are noted above the SD bars. Bottom, Linear regression plot of initial measurement and subsequent measurement for all time intervals, showing tight correlation for all plaque sizes. The slope of the line approaches unity (0.98) with a correlation coefficient $R^{2}=0.89$.

plaques from one imaging session to another. After carefully evaluating $>300$ pairs of plaques, we were able to find only 14 clear examples of marked growth or resolution (i.e., a change in size by $40 \%$ ). Figure 6 shows examples of plaques from a volumerendered stack of images of the same region of cortex, obtained $104 \mathrm{~d}$ apart, showing the same four plaques (in red) as well as the fluorescein angiogram (green). Qualitative and quantitative analyses show that two of the plaques have grown substantially $(\sim 50 \%)$, one has become substantially smaller (by $>40 \%$ ), and one has not changed size at all. These data show that, within the same region and during the same imaging sessions, some plaques appear to grow and others shrink. No tendency for large plaques to shrink or small plaques to grow (i.e., a correlation between initial diameter and percentage change) was evident in the data. Technical issues such as thioflavine S concentration or power at the focal plane cannot account for some getting larger and others getting smaller within a region as small as a single threedimensional field; the most parsimonious explanation is that, in these instances, plaque size is changing.

With increasing age, the number of thioflavine S-positive plaques in the cortex is known to increase (Hsiao et al., 1996; Irizarry et al., 1997). Thus, when re-imaging a volume of the cortex, one would expect to occasionally find new plaques within the imaging volume. Again, we used rigorous criteria to ensure that the appearance of a new plaque did not simply reflect slightly better signal-to-noise characteristics in a second imaging session than in the first, or a greater depth of imaging, or a slightly different imaging volume. Compelling examples, in which a new
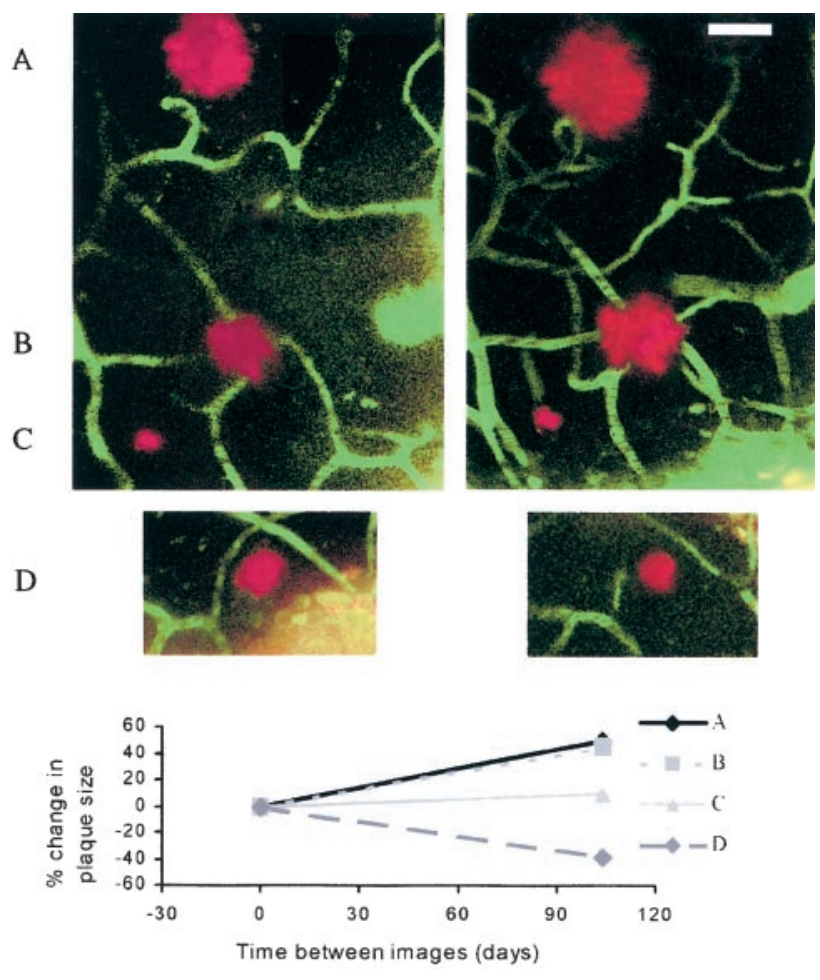

Figure 6. A subpopulation of plaques change size over time. The images are two-channel volume-rendered stacks of thioflavine S plaques $(r e d)$ and fluorescein angiograms (green) taken from the same animal at the initial imaging session (left images) and $104 \mathrm{~d}$ later (right images). Four clearly imaged plaques can be seen in these volumes, labeled $A-D$. The autofluorescence of the dura appears at the upper edge of the volume stacks and appears slightly different in the images here and in Figure 6 because the image stacks are not exactly coincident at their initial depth. The graph below represents the percentage change in diameter for each plaque. The plaques labeled $A$ and $B$ increase in size by $\sim 50 \%$, plaque $C$ remains the same size, and plaque $D$ decreases by $40 \%$. Scale bar, $20 \mu \mathrm{m}$.
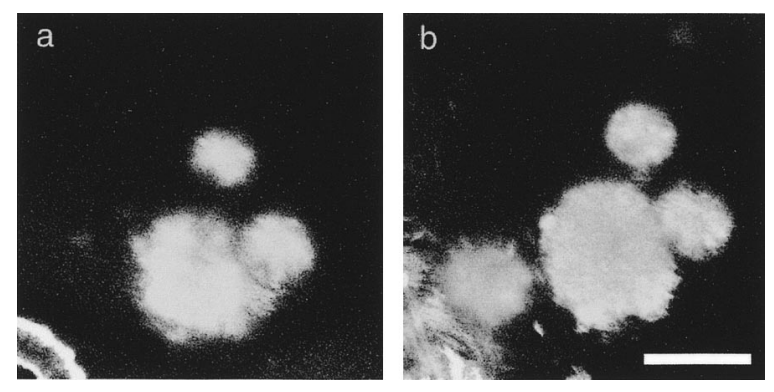

Figure 7. Appearance of a novel plaque in the imaged region. $a$, Volume rendering of a set of three plaques during an initial imaging session. $b$, Volume rendering of the same region imaged $64 \mathrm{~d}$ later, showing the initial plaques joined by a novel thioflavine S-positive plaque. The fibrous autofluorescence at bottom left is dura mater. Scale bar, $50 \mu \mathrm{m}$.

plaque appeared in a volume that had been previously imaged, occurred nonetheless. Figure 7 shows a dramatic example of a field with three well defined, characteristic plaques at the first imaging session and four at the second imaging session $64 \mathrm{~d}$ later.

The in vivo longitudinal data noted above suggest that the average plaque diameter does not vary with age. To examine this conclusion using traditional histological analyses in $\mathrm{Tg} 2576$ mice, we examined thioflavine S-stained sections from mice either 12 
months $(n=3)$ or 22 months $(n=3)$ of age using a Bioquant image analysis system (Hyman et al., 1993). The average number of plaques in the cortex increases nearly sixfold over this 10 month period, from $2.3 \pm 1.4$ to $13.7 \pm 4.3$ plaques $/ \mathrm{mm}^{2}$ (mean \pm $\mathrm{SD}, p<0.05)$. The size distribution of plaque diameters does not change appreciably between 12 and 22 months, from $18.1 \pm 17.8$ to $21.4 \pm 16.2 \mu \mathrm{m}$ ( $p>0.05$, not significant). These crosssectional data are consistent with in vivo measurements, suggesting that plaque size is stable over an extended period of time. Together with our in vivo measures, these data are consistent with a model in which plaques are formed, reach their maximal size rather quickly, and then stop growing.

\section{DISCUSSION}

In this report, we demonstrate the successful imaging of senile plaques in living transgenic animals using in vivo multiphoton laser scanning microscopy. No other imaging approach has the resolution necessary to observe these Alzheimer's disease-like lesions. Thus, very little is known about the natural history of these deposits in the living brain. Multiphoton microscopy permits high-resolution imaging of living tissue with minimal photodamage or toxicity. Imaging through an intact skull window allows chronic, in vivo brain imaging over periods of days to months. Using thioflavine $\mathrm{S}$, a sensitive and specific fluorescent reporter for the dense-core subset of senile plaques, we were able to follow a population of identified plaques in living transgenic mice over time. Over periods ranging from days to as long as 5 months, the size and morphology of individual thioflavine S-positive plaques remain remarkably stable. Our results suggest that these plaques, once formed, are quickly stabilized. If continual deposition of insoluble $\mathrm{A} \beta$ is assumed, this size constancy confirms the predictions of the dynamic feedback hypothesis (Hyman et al., 1995; Cruz et al., 1997; Urbanc et al., 1999a,b). Stability of plaque size over time is also in accord with the predictions of a rapid time-limited aggregation of peptide, reflecting a transient increase in local peptide concentration above the threshold for nucleation-dependent growth (Jarrett and Lansbury, 1993; Esler et al., 1996). An intriguing avenue of further research is to understand the mechanism and time course of the initial plaque formation. This imaging technique may provide the means for addressing this issue in the future. We were also able to observe shrinkage of individual plaques for the first time, confirming the hypothesis that, to some extent, plaques are in a dynamic equilibrium with their environment. This raises the possibility that clearance of plaques that have heretofore been considered insoluble may be possible with appropriately targeted therapeutics.

The current observations also raise the new question of why plaques stop growing. We speculate that glia promptly respond to the presence of an abnormal deposit in the neuropil either by surrounding it or by phagocytosis. In Alzheimer's disease and in the transgenic models that we have studied, glia may play an active role in halting plaque growth primarily by surrounding the deposits (Frautschy et al., 1998). Recent experiments using immunization with amyloid- $\beta$ in another transgenic model of Alzheimer's disease suggest that microglia can phagocytose antibody-bound amyloid deposits (Schenk et al., 1999). We hypothesize that glial interaction with amyloid deposits may be the biological mechanism responsible for the "dynamic feedback" postulated in the theoretical model (Hyman et al., 1995; Cruz et al., 1997; Urbanc et al., 1999a,b), stabilizing the size of plaques and preventing continued growth. Further experiments that spe- cifically inhibit the activity of microglia should address this hypothesis directly.

Our current experiments do not directly answer questions about the portion of amyloid- $\beta$ deposits that are not thioflavine $\mathrm{S}$ positive. Insofar as thioflavine $\mathrm{S}$-negative amyloid- $\beta$ deposits are generally less compact and may not be cross-linked, one might expect them to be more dynamic. Similarly, our data suggest that the vast majority of thioflavine $\mathrm{S}$ plaques, once formed, do not grow in size and also do not resolve over the timespan that we studied. Nonetheless, we cannot rule out the possibility that rare plaques do resolve spontaneously. We did fail to re-image some plaques, but after using conservative criteria regarding technical issues that might have contributed to failure to re-image, we were unable to identify any unequivocal examples of clearance.

This study demonstrates the ability to observe amyloid plaques chronically in a living brain using in vivo multiphoton microscopy that provides resolution on the order of confocal microscopy at unprecedented depths with negligible tissue damage. This technique will allow longitudinal studies of individual animals subjected to experimental manipulations and should be particularly powerful for the investigation of therapeutics targeted at clearing amyloid. Likewise, development of fluorophores that identify other pathological features, including the surrounding non-thioflavine S-staining amyloid- $\beta$, holds the promise for substantial advances in understanding brain pathophysiology in transgenic models of disease. In principle, this same approach could be used to diagnose and follow amyloid- $\beta$ deposition in the human brain in Alzheimer's disease.

\section{REFERENCES}

Cruz L, Urbanc B, Buldyrev SV, Christie R, Gomez-Isla T, Havlin S, McNamara M, Stanley HE, Hyman BT (1997) Aggregation and disaggregation of senile plaques in Alzheimer disease. Proc Natl Acad Sci USA 94:7612-7616.

Denk W, Strickler JH, Webb WW (1990) Two-photon laser scanning fluorescence microscopy. Science 248:73-76.

Esler WP, Stimson ER, Ghilardi JR, Vinters HV, Lee JP, Mantyh PW, Maggio JE (1996) In vitro growth of Alzheimer's disease betaamyloid plaques displays first-order kinetics. Biochemistry 35:749-757.

Frautschy SA, Yang F, Irrizarry M, Hyman B, Saido TC, Hsiao K, Cole GM (1998) Microglial response to amyloid plaques in APPsw transgenic mice. Am J Pathol 152:307-317.

Hilbich C, Kisters-Woike B, Reed J, Masters CL, Beyreuther K (1991) Aggregation and secondary structure of synthetic amyloid beta A4 peptides of Alzheimer's disease. J Mol Biol 218:149-163.

Hsiao K, Chapman P, Nilsen S, Eckman C, Harigaya Y, Younkin S, Yang F, Cole G (1996) Correlative memory deficits, A $\beta$ elevation, and amyloid plaques in transgenic mice. Science 274:99-102.

Hyman BT, Tanzi RE, Marzloff K, Barbour R, Schenk D (1992) Kunitz protease inhibitor-containing amyloid beta protein precursor immunoreactivity in Alzheimer's disease. J Neuropathol Exp Neurol 51:76-83.

Hyman BT, Marzloff K, Arriagada PV (1993) The lack of accumulation of senile plaques or amyloid burden in Alzheimer's disease suggests a dynamic balance between amyloid deposition and resolution. J Neuropathol Exp Neurol 52:594-600.

Hyman BT, West HL, Rebeck GW, Buldyrev SV, Mantegna RN, Ukleja M, Havlin S, Stanley HE (1995) Quantitative analysis of senile plaques in Alzheimer disease: observation of log-normal size distribution and molecular epidemiology of differences associated with apolipoprotein E genotype and trisomy 21 (Down syndrome). Proc Natl Acad Sci USA 92:3586-3590.

Irizarry MC, McNamara M, Fedorchak K, Hsiao K, Hyman BT (1997) APPSw transgenic mice develop age-related A beta deposits and neuropil abnormalities, but no neuronal loss in CA1. J Neuropathol Exp Neurol 56:965-973.

Jarrett JT, Lansbury Jr PT (1993) Seeding "one-dimensional crystallization" of amyloid: a pathogenic mechanism in Alzheimer's disease and scrapie? Cell 73:1055-1058.

Kelenyi G (1967) Thioflavin S fluorescent and Congo red anisotropic 
stainings in the histologic demonstration of amyloid. Acta Neuropathol (Berl) 7:336-348.

Mirra SS, Heyman A, McKeel D, Sumi SM, Crain BJ, Brownlee LM, Vogel FS, Hughes JP, van Belle G, Berg L (1991) The Consortium to Establish a Registry for Alzheimer's Disease (CERAD). Part II. Standardization of the neuropathologic assessment of Alzheimer's disease. Neurology 41:479-486.

Schenk D, Barbour R, Dunn W, Gordon G, Grajeda H, Guido T, Hu K, Huang J, Johnson-Wood K, Khan K, Kholodenko D, Lee M, Liao Z, Lieberburg I, Motter R, Mutter L, Soriano F, Shopp G, Vasquez N, Vandevert C, Walker S, Wogulis M, Yednock T, Games D, Seubert P (1999) Immunization with amyloid-beta attenuates Alzheimerdisease-like pathology in the PDAPP mouse. Nature 400:173-177.

Schmidt ML, Robinson KA, Lee VM, Trojanowski JQ (1995) Chemical and immunological heterogeneity of fibrillar amyloid in plaques of
Alzheimer's disease and Down's syndrome brains revealed by confocal microscopy. Am J Pathol 147:503-515.

Svoboda K, Denk W, Kleinfeld D, Tank DW (1997) In vivo dendritic calcium dynamics in neocortical pyramidal neurons. Nature 385:161-165.

Urbanc B, Cruz L, Buldyrev SV, Havlin S, Hyman BT, Stanley HE (1999a) Dynamic feedback in an aggregation-disaggregation model. Phys Rev E 60:2120-2126.

Urbanc B, Cruz L, Buldyrev SV, Havlin S, Irizarry MC, Stanley HE, Hyman BT (1999b) Dynamics of plaque formation in Alzheimer's disease. Biophys J 76:1330-1334.

Vonsattel JP, Myers RH, Hedley-Whyte ET, Ropper AH, Bird ED, Richardson Jr EP (1991) Cerebral amyloid angiopathy without and with cerebral hemorrhages: a comparative histological study. Ann Neurol 30:637-649. 